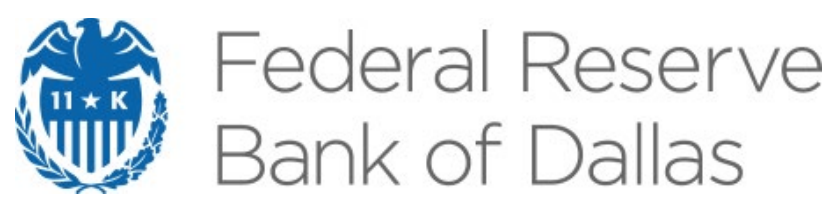

\title{
Mildly Explosive Dynamics in U.S. Fixed Income Markets
}

Silvio Contessi, Pierangelo De Pace and Massimo Guidolin

\section{Globalization Institute Working Paper 324}

Research Department

https://doi.org/10.24149/gwp324r1

Working papers from the Federal Reserve Bank of Dallas are preliminary drafts circulated for professional comment. The views in this paper are those of the authors and do not necessarily reflect the views of the Federal Reserve Bank of Dallas or the Federal Reserve System. Any errors or omissions are the responsibility of the authors. 


\title{
Mildly Explosive Dynamics in U.S. Fixed Income Markets
}

\author{
Silvio Contessi ${ }^{\dagger}$, Pierangelo De Pace ${ }^{\ddagger}$ and Massimo Guidolin§
}

August 2017

Revised: February 4, 2019

\begin{abstract}
We use a recently developed right-tail variation of the Augmented Dickey-Fuller unit root test to identify and date-stamp periods of mildly explosive behavior in the weekly time series of eight U.S. fixed income yield spreads between September 2002 and April 2018. We find statistically significant evidence of mildly explosive dynamics in six of these spreads, two of which are short/medium-term mortgage-related spreads. We show that the time intervals characterized by instability that we estimate from these yield spreads capture known episodes of financial and economic distress in the U.S. economy. Mild explosiveness migrates from short-term funding markets to mediumand long-term markets during the Great Financial Crisis of 2007-09. Furthermore, we statistically validate the conjecture, originally suggested by Gorton (2009a b), that the initial panic of 2007 migrated from segments of the ABX market to other U.S. fixed income markets in the early phases of the financial crisis.
\end{abstract}

JEL Classifications: G01, G12, C58, C44

Keywords: Finance, investment analysis, fixed income markets, yield spreads, mildly explosive behavior.

\footnotetext{
*We are grateful to Michael Owyang, Michael McCracken, David Rapach, William Dupor, Peter Reinhard Hansen, Riccardo DiCecio, Alma Bezares Calderón, Hisam Sabouni, and the participants of several conferences for many constructive suggestions and remarks. We also thank George William Abele for excellent research assistance.

†Silvio Contessi, Monash Business School, Department of Banking and Finance, P.O. Box 197, Caulfield East, VIC 3145 , Australia, silvio.contessi@monash.edu, phone: +61 399034956.

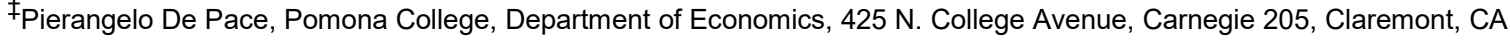
91711, USA, pierangelo.depace@pomona.edu, phone: +19096218744.

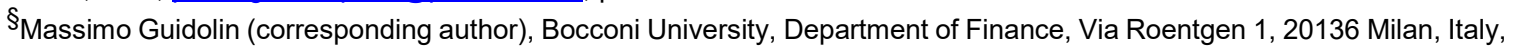
and Baffi CAREFIN Centre, Massimo.Guidolin@unibocconi.it, phone: +39 0258363505.
} 


\section{Introduction}

Researchers and practitioners view U.S. fixed income markets as the epicenter of the Great Financial Crisis of 2007-09. Security yields in these markets are generally used to construct yield spreads that are widely adopted in theoretical and empirical modeling in macroeconomics and finance and as measures of risk in asset management. Often, yield spreads offer investors a clearer picture of the underlying risk-return trade-offs than the individual yields (interest rates) that are used to construct them. Yield spreads can be especially informative of the channels through which asset prices affect (or are related to) the real economy, as their magnitude tends to vary following or anticipating the business cycle 1 As extensively documented in the empirical literature, many spreads tend to suddenly spike at times of financial distress $:^{2}$ Consequently, understanding the dynamics of risk and of the risk premia incorporated in the prices of bonds and in the corresponding spreads has practical implications for policymakers, finance practitioners, and investors. The ability to identify the particular market segments in which risk premia exhibit unstable dynamic behavior at times of crisis may allow policy makers to better target and calibrate their interventions, and may complement traditional early-warning indicators of impending recessions (Huang et al., 2017). By possibly understanding and forecasting how such unstable dynamics may migrate across markets and sectors of the economy, policymakers would be able to evaluate the degree of insulation of individual markets from aggregate and systemic shocks. Moreover, changes in the evolution of risk premia may suggest to investors alternative diversification approaches. All these considerations explain the recent interest of economists, econometricians, and applied mathematicians in investing in and forecasting the relationships involving yield spreads.

In this paper, we propose an empirical exercise characterized by a twofold objective: (i) the identification of the segments in the U.S. fixed income markets that were the core ground of the Great Financial Crisis - i.e., the segments where financial distress appeared first; and (ii) the description of how the 2007 financial turmoil developed and spread across markets in the subsequent two years. We do so by examining the weekly time series of eight yield spreads derived from a variety of risky instruments traded in U.S. fixed income markets - which we treat as distinct asset classes - between the second half of 2002 and April 2018. The traded yields include the 3-month London interbank offered rate (LIBOR) on unsecured deposits, the 3-month unsecured financial and asset-backed commercial paper (ABCP) rate,

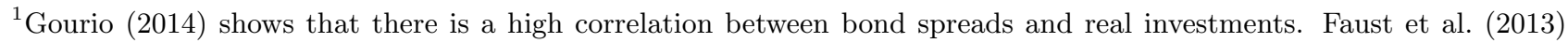
report that some credit spreads improve the forecast accuracy of real-time economic activity. Hollander and Liu (2016) document significant widening of several credit spreads during the most recent U.S. recessions. Recchioni and Tedeschi (2017) discuss the relationship between government bond yields and the macroeconomy. Fanelli (2017) shows the relevance of credit spreads volatilities for interest rate curve modeling and asset pricing.

${ }^{2}$ See Muir (2017) and Krishnamurty and Muir (2017) for a cross-country perspective, and Guidolin and Tam (2013) for a specific view on the Great Financial Crisis in the U.S.
} 
the 1-year yield on adjustable-rate mortgages (1-year ARM), the 5-year 5/1 hybrid adjustable-rate mortgages (5-year ARM), the 5-year Aaa private-label commercial mortgage-backed securities (CMBS) rate, the yields on 20-year Moody's Baa-rated and Aaa-rated corporate bonds, the 20-year Bloomberg Fair Value U.S. Dollar Composite Bbb-rated and Aa-rated corporate bond rates, and the 30-year conventional fixed-rate mortgage-backed securities (MBS) yield. Given the relevance of the U.S. mortgage market in the 2007-09 crisis (Gorton, 2009a), we include four series from which mortgage-related risk premia are typically constructed. Furthermore, given that each spread is computed as the difference of two yields of the same maturity (3 months, 1 year, 5 years, 20 years, and 30 years), risk premia associated with mismatched durations of the underlying assets are not included the dataset. Because they are computed from Treasury yields or corporate yields on assets with low(er) risk profiles, these eight yield spreads contain information about the credit and (il)liquidity risk factors priced in U.S. asset markets.

This paper builds upon two strands of literature. The first strand consists of recent empirical work conducted on U.S. yield spreads and their relation with macroeconomic variables and fluctuations (see, for example, Guidolin and Tam, 2013; Contessi et al., 2014; Hollander and Liu, 2016; Del Negro et al., 2017; Clark and Baccar, 2018). The second strand relates to research on optimal methods developed to detect episodes of contagion and/or bubbles in asset price data and to studies about their transmission across sectors, industries, or economies (see Forbes and Rigobon, 2001, 2002; Dungey et al., 2005; Pesaran and Pick, 2007; Hayford and Malliaris, 2005; Kürüm et al., 2018) ? $^{3}$

In our paper, we adopt a testing and date-stamping technique, initially formulated by Phillips and Yu (2011) and later refined in Phillips et al. (2015), to identify the periods over which the eight yield spreads in the sample exhibit unstable dynamics - i.e., what we shall formally define as mildly explosive behavior. From a statistical point of view, this approach is based on a recursive, rolling right-tail variation in the implementation of the Augmented Dickey-Fuller $(A D F)$ unit root test in which, under the alternative hypothesis, the time-series process under investigation exhibits (at least locally) a root larger than one. In its original formulation, such an empirical strategy allows for the detection (and the date-stamping of both origination and termination) of bubbles in the time series of the prices of an asset of interest. For example, Phillips and $\mathrm{Yu}(2011)$ interpret the Baa-Aaa corporate yield spread as a measure of the price of risk in bond markets. To the extent to which this interpretation is reasonable, a period of mildly explosive behavior in the time series of such a spread, if associated with a widening spread, can be viewed

\footnotetext{
${ }^{3}$ There are interesting attempts at merging the two strands of literature. For instance, Recchioni and Tedeschi (2017) develop a simple and analytically tractable common stochastic, mean-reverting volatility model in continuous time that captures yield dynamics. They exploit the empirically high correlation between the estimated volatility parameters and the instability in bond yields to build an early-warning indicator of significant instabilities, similar in spirit to what we apply in our work. They report that their indicator identifies three bubbles that anticipate three major episodes of instability - i.e., the sub-prime mortgage crisis, the collapse of Lehman Brothers, and the European sovereign debt crisis.
} 
as a bubble in the price of risk in the underlying market. More broadly, also depending on the specific context of application, this strategy can be used to identify episodes of exuberance and collapse, as well as structural breaks, periods of regime change, or instances of panic and turmoil in a given market. We show that the mildly explosive behavior that we detect in the time series of yield spreads in our sample, especially in the sub-samples in which the spreads exhibit an upward sloping trajectory, corresponds to well-known episodes of turmoil in the U.S. markets, which therefore - using the techniques illustrated in this paper - could have been detected, monitored, and partially predicted in real time (see Huang et al., 2017). Additionally, we find evidence of mildly explosive behavior in six out of the eight yield spreads under investigation. Two of these six spreads are short/medium-term mortgage-related spreads. We show that the strength of such unstable dynamics peaked between August 2007 and January 2009 and occurred, sequentially, first in short-term funding and later in medium- and long-term markets, which represents a clear and plausible migration pattern.

In the last part of the paper, we formally investigate the conjecture, originally proposed by Gorton $(2009 \mathrm{a} b)$, that the collapse of the synthetic collateralized debt market based on sub-prime residential mortgages could have been one of the main reaction chambers of the Great Financial Crisis - i.e., the epicenter of a panic/turmoil episode that triggered a chain reaction that spread across all U.S. fixed income markets. A recent strand of literature straddling financial economics and applied econometrics has investigated contagion phenomena and systemic risk with applications to bond yields (see Recchioni and Tedeschi, 2017), with reference to the European sovereign debt crisis. Therefore, we use statistical methods to explore the possibility that the financial panic of 2007 initially migrated from specific segments of the market for sharing and allocating (correlation) risks in sub-prime loans (through the trading of ABX indices) to other fixed income markets. Seen through the lens of a model of bank/financial runs with sunspot equilibria, the drop in the ABX indices that occurred in 2007 may have acted as a focal shock that favored the emergence of a (shadow) bank-run equilibrium consistent with the financial run mechanism described in Diamond and Dybig (1983). We provide statistical support to Gorton (2009a b)'s conjecture through the identification of a panic transmission pattern that goes from the market of subprime residential mortgages to some other key U.S. fixed income yields.

The rest of this paper is structured as follows. Section 2 summarizes our methodology. Section 3 describes our data. Section 4 discusses the results and their interpretation. Section 5 revisits the argument made in Gorton (2009a b) in the context that we propose. Section 6 concludes. 


\section{Research Methodology}

Failing to recognize unstable dynamics in time-series data, in real, or almost real, time, has potentially serious implications for both policy making and investment strategies. Despite the frequent discussions on the difficulties in the identification of asset bubbles (Bernanke, 2002), policy makers often advocate increasing interest rates to curb excessive growth of asset prices or, more generally, to prevent financial instability (Esther, 2016). In fact, since the Great Financial Crisis, several central banks followed the lead of the Federal Reserve and started developing systems of monitoring for financial markets, as documented in Adrian et al. (2015). Despite the fact that the identification of bubbles is, in general, not an easy task to accomplish, the recent studies that we follow have developed tests for the empirical detection of bubbles in price data based on a combination of theoretical predictions and time-series estimation techniques. Derived from asset pricing theory, their main idea is that, if a bubble develops in an asset market, prices should inherit and exhibit, at least locally and for a limited time, an explosive dynamic behavior.

Bubble detection strategies are recently described, for example, in Phillips and Yu (2011) and Phillips et al. (2015). Their econometric methodology can detect bubbles in the data and date-stamp their occurrence. Their tests use recursive and rolling right-tail variations of the $A D F$ unit root test in which, under the null hypothesis, the time series of interest has a unit root and, under the alternative hypothesis, the observed time series has, at least locally, a root larger than one - i.e., technically, it is a mildly explosive stochastic process. If the null hypothesis of their tests is rejected, one can then estimate the origination and termination of a bubble or of multiple bubbles. Phillips et al. (2015) show that a specific version of their procedure (based on recursive and flexible windows) can be used, under general regularity conditions, as a date-stamping strategy able to consistently estimate the origination and termination of bubbles in long historical time series. Through Monte Carlo simulations, they also demonstrate that their strategy outperforms the approach initially proposed in Phillips and Yu (2011). In particular, they argue that their test significantly improves the discriminatory power and leads to non-negligible power gains when multiple bubbles are present in the data.

Figure 1 describes the steps in the procedure that we adopt to detect and date-stamp periods of mildly explosive behavior in the yield spreads in the sample. Details are given in the following subsections.

\subsection{Testing for the Presence of Mildly Explosive Behavior}

The first step of the procedure is a test used to detect mild explosiveness in a time series of interest.

(a) The testing strategy is based on the estimation of the following reduced-form equation, 


$$
y_{t}=\mu+\delta y_{t-1}+\sum_{i=1}^{p} \phi_{i} \Delta y_{t-i}+\varepsilon_{t},
$$

where $y_{t}$ is the time series of interest, $\mu$ is an intercept, $p$ is the maximum number of lags, and $\varepsilon_{t}$ is the error term. Testing for mildly explosive behavior is based on a right-tail variation of the standard $A D F$ unit root test.

(b) We follow Phillips et al. (2015) and consider the hypotheses, $H_{0}: \delta=1$ vs $H_{1}: \delta>1$. We normalize the original sample interval of $T$ observations to the compact $[0,1]$. The $\delta$ coefficient, estimated by ordinary least squares over the (normalized) sample $\left[r_{1}, r_{2}\right] \subseteq[0,1]$, and its corresponding $A D F$ test statistic are denoted by $\delta_{r_{1}, r_{2}}$ and $A D F_{r_{1}, r_{2}}$, respectively. We define the (fractional) window size of the regression as $r_{w}=r_{2}-r_{1}$. The Generalized Supremum Augmented Dickey-Fuller $(G S A D F)$ test is derived from a recursive procedure in which the $A D F$ test statistic is calculated over (overlapping) rolling windows of increasing sizes and moving starting points (i.e., over a forward rolling and expanding sample). Each estimation in this recursive approach produces an $A D F$ test statistic. The $G S A D F$ test statistic is the supremum $A D F_{r_{1}, r_{2}}$ statistic over all possible windows,

$$
G S A D F\left(r_{0}\right)=\sup _{\substack{r_{2} \in\left[r_{0}, 1\right] \\ r_{1} \in\left[0, r_{2}-r_{0}\right]}}\left\{A D F_{r_{1}, r_{2}}\right\}
$$

where $r_{0}$ is the smallest sample window width fraction (which initializes the computation of the test statistic, in our paper set to $10 \%$ ) and 1 is the largest window width fraction (corresponding to the full sample size) in the recursion. The recursion mechanism is represented graphically in Figure 2 .

(c) The relevant critical values are the simulated as follows.

(i) We generate a random sample of $T$ observations based on a null model, which, as in Phillips et al. (2015), is a random walk process with an asymptotically negligible drift,

$$
y_{t}=d T^{-\eta}+\theta y_{t-1}+e_{t}, e_{t} \sim N\left(0, \sigma^{2}\right), \theta=d=\eta=1,
$$

where $\eta$ is a localizing coefficient that controls the magnitude of the drift as $T \longrightarrow \infty$ and $e_{t}$ is a normal error term.

(ii) We recursively estimate equation (1) by ordinary least squares, using the recursion that we describe in Figure 2, over the sample generated by the null model, and then store the resulting $G S A D F$ test statistic. 
(iii) We repeat first and second steps 1,000 times.

(iv) We calculate the $90 \%$ quantile of the distribution of the GSADF test statistic produced from these 1,000 simulations. This quantile is used to test the null of a unit root against the alternative of a mildly explosive process. The simulation output includes the p-value for the computed test statistic, here defined as

$$
p(\widehat{\tau})=\frac{1}{1,000} \sum_{j=1}^{1,000} I\left(\tau_{j}>\widehat{\tau}\right),
$$

where $\widehat{\tau}$ is the sample $G S A D F$ test statistic, $I(\cdot)$ is an indicator function such that

$$
I\left(\tau_{j}>\widehat{\tau}\right)= \begin{cases}1 & \text { if } \tau_{j}>\widehat{\tau} \\ 0 & \text { if } \tau_{j} \leqslant \widehat{\tau}\end{cases}
$$

and $\left\{\tau_{j}\right\}_{j=1}^{1,000}$ is the sequence of simulated $G S A D F$ test statistics.

\subsection{Date-Stamping Periods of Mildly Explosive Behavior}

The procedure outlined in Figure 1 then proceeds to date-stamp periods of mildly explosive behavior.

(d) If the null hypothesis of the $G S A D F$ test is rejected, a similar procedure as in the previous subsection can be used, under general regularity conditions, as a date-stamping strategy to consistently estimate origination and termination of periods of mildly explosive behavior. We implement a recursive Supremum $A D F$ test on backward expanding samples, using an algorithm specular to the one that we have described in the previous subsection. The end point, which now moves backwards, of each sample is fixed at $r_{2}$ and the start point is allowed to vary from 0 to $r_{2}-r_{0}$. For each $r_{2}$, we obtain a sequence of $A D F$ test statistics, $\left\{A D F_{r_{1}, r_{2}}\right\}_{r_{1} \in\left[0, r_{2}-r_{0}\right]}$, and a Backward Supremum $A D F$ test statistic, defined as the supremum value of the $A D F$ test statistic sequence over this interval,

$$
B S A D F_{r_{2}}\left(r_{0}\right)=\sup _{r_{1} \in\left[0, r_{2}-r_{0}\right]}\left\{A D F_{r_{1}, r_{2}}\right\}
$$

(e) Based on the sequence of test statistics, estimates of beginning $\left(\widehat{r}_{e}\right)$ and termination $\left(\widehat{r}_{f}\right)$ of a period of mildly explosive behavior (as fractions of the full sample) are given by

$$
\widehat{r}_{e}=\inf _{r_{2} \in[0,1]}\left\{r_{2}: B S A D F_{r_{2}}\left(r_{0}\right)>c v_{r_{2}}^{\beta_{T}}\right\}
$$


and

$$
\widehat{r}_{f}=\inf _{r_{2} \in\left[\widehat{r}_{e}, 1\right]}\left\{r_{2}: B S A D F_{r_{2}}\left(r_{0}\right)<c v_{r_{2}}^{\beta_{T}}\right\}
$$

where $c v_{r_{2}}^{\beta_{T}}$ is the $100\left(1-\beta_{T}\right) \%$ critical value of the $B S A D F$ test statistic based on $\operatorname{Tr}_{2}$ observations and $\beta_{T}$ is a real number between 0 and 1 indicating the level of significance of the test. In other words, the origination date is the observation at which the $B S A D F$ statistic exceeds the critical value of the $B S A D F$ statistic. Similarly, the termination date is the observation at which the $B S A D F$ statistic falls below the critical value of the $B S A D F$ statistic. The $G S A D F$ test and the $B S A D F$ test statistics are related to each other - i.e.,

$$
G S A D F\left(r_{0}\right)=\sup _{r_{2} \in\left[r_{0}, 1\right]}\left\{B S A D F_{r_{2}}\left(r_{0}\right)\right\}
$$

\subsection{Migration of Mildly Explosive Behavior}

The steps that we represent in Figure 1 are finally completed in this subsection. The reduced-form algorithm to test migration of mildly explosive behavior from one series $X_{t}$ to another series $Y_{t}$ is originally described in Phillips and $\mathrm{Yu}(2011)$.

(f) Let $\theta_{X}(\tau)$ be the coefficient of an autoregressive model with an intercept term, for the time series $\left\{X_{t}\right\}_{t=1}^{\tau=T r}$ with $r \in\left[r_{0}, 1\right] . \quad \theta_{X}(\tau)$ can be estimated by ordinary least squares as $\widehat{\theta}_{X}(\tau)$ over a recursively increasing window with a fixed starting date that occurs as early as practically feasible in the sample. We define $\theta_{Y}(\tau)$ and $\widehat{\theta}_{Y}(\tau)$ similarly. By allowing for time variation in $\theta_{X}(\tau)$, we try to capture possible structural changes in the coefficient(s) originating from episodes of turmoil, panic, or market exuberance. Our goal is to test the presence of migratory effects in the dynamics of a second time series, $Y_{t}$. The intuition is that, when mild explosiveness reaches its peak in $X_{t}$ (a local maximum in the sequence of $B S A D F$ test statistics), we can test for its transmission to $Y_{t}$. Under the alternative of migration, mildly explosive behavior emerges in $Y_{t}$ as it fades away in $X_{t}$. From a modeling point of view, the null generating mechanism of $Y_{t}$ has a recursive autoregressive coefficient, $\theta_{Y}(\tau)$, that transitions from a unit root to a mildly explosive root and that is negatively associated with the corresponding recursive autoregressive coefficient for $X_{t}, \theta_{X}(\tau)$.

(g) Suppose that the date-stamping procedure that we have described has identified mildly explosive behavior in $X_{t}$ between $\widehat{\tau}_{e X}=T \widehat{r}_{e X}$ and $\widehat{\tau}_{f X}=T \widehat{r}_{f X}$ and in $Y_{t}$ between $\widehat{\tau}_{e Y}=T \widehat{r}_{e Y}$ and $\widehat{\tau}_{f Y}=$ 
$T \widehat{r}_{f Y}$. Also assume that the two sequences of $B S A D F$ statistics for $X_{t}$ and $Y_{t}$ peak at times $\widehat{\tau}_{\rho X}=T \widehat{r}_{\rho X}$ and $\widehat{\tau}_{\rho Y}=T \widehat{r}_{\rho Y}$, respectively, and that $\widehat{r}_{\rho Y}>\widehat{r}_{\rho X}$. Let $m=\widehat{\tau}_{\rho Y}-\widehat{\tau}_{\rho X}=T \widehat{r}_{\rho Y}-T \widehat{r}_{\rho X}$ be the number of observations in the interval $\left(\widehat{\tau}_{\rho X}, \widehat{\tau}_{\rho Y}\right]$. Phillips and $\mathrm{Yu}(2011)$ show that the notion of migration that we have described can be detected by first estimating the regression,

$$
\left[\widehat{\theta}_{Y}(\tau)-1\right]=\beta_{0}+\beta_{1}\left[\widehat{\theta}_{X}(\tau)-1\right] \frac{\tau-\widehat{\tau}_{\rho X}}{m}+\text { error, with } \tau=T \widehat{r}_{\rho X}+1, \ldots, T \widehat{r}_{\rho Y}
$$

over a sample covering the period of collapse in $X_{t}$ and the coincident emergence of explosiveness in $Y_{t}$, and then by testing $H_{0}: \beta_{1}=0$ vs $H_{1}: \beta_{1}<0$. An asymptotically conservative and consistent test for this hypothesis is based on the standard normally distributed test statistic,

$$
Z_{\beta}=\frac{\widehat{\beta}_{1}}{L(m)}, \text { where } \frac{1}{L(m)}+\frac{L(m)}{T^{\epsilon}} \longrightarrow 0, \text { as } T \longrightarrow \infty \text { for any } \epsilon>0
$$

for some slowly varying function $L(m)$, such as $a \log _{10}(m)$, with $a>0$ and $m=O(T)$.

\section{Data}

The empirical methods described in the previous section are applied to the identification, if any, of periods of explosive behavior in the eight time series in our sample. The objective is to determine the beginning and the end of episodes of unstable dynamics, and to test for their migration across U.S. fixed income markets, using data concerning eight interest rate spreads of interest. The series are collected from Bloomberg and organized in a sample of weekly observations, as typical in the literature. We consider a sample that spans between the week of September 20, 2002 and the week of April 20, 2018, for a total of 814 weekly observations. However, as we describe later, some spreads may cover different time periods between these two dates, depending on data availability. These eight spreads exhibit some degree of heterogeneity that depends on the fixed income markets to which they refer, the maturity of the underlying securities, and whether or not they were affected by specific policy measures implemented by the Federal Reserve Bank, the United States Treasury, or the Federal Deposit Insurance Corporation; or by other policy interventions that occurred during the Great Financial Crisis. We will refer to these spreads using a number and a descriptor, both reported in bold in the next paragraphs.

Spread 1 (3-Month LIBOR-OIS) is the 3-month LIBOR on unsecured deposits relative to the overnight indexed swap (OIS) rate. The 3-month LIBOR is the interest rate that banks face when they borrow unsecured funds on the interbank market with a 3-month maturity. The OIS rate is the fixed 
interest rate that a bank receives in 3-month swaps between a fixed rate and a compound interest payment on a notional amount to be determined with reference to the effective federal funds rate. The LIBOROIS spread is widely perceived as an indicator of tensions in money markets, a measure of health of the banking system, and as an index of risk and liquidity in the money market. It is a standard indicator of liquidity premium of widespread use and was possibly affected by swap arrangements among central banks during the Great Financial Crisis. While there are legitimate concerns that, after the LIBOR scandal emerged in 2008, the use of the LIBOR for analysis may require caution, recent research suggests that LIBOR rates still remain a good measure of financial distress ${ }^{4}$

Spread 2 (3-Month ABCP-Treasury) is the yield on 3-month ABCP relative to Treasury Bills of the same maturity. ABCP experienced a dramatic drop in transaction volumes during the financial crisis, a shortage that made this spread particularly reflective of both liquidity and credit risk. Later on, still during the crisis, this spread became a direct target of the Asset-Backed Commercial Paper Money Market Mutual Fund Liquidity Facility (which began operations on September 22, 2008, and was closed on February 1, 2010) and the Commercial Paper Funding Facility (which was announced on October 7, 2008, began purchases of commercial paper on October 27, 2008, and was closed on February 1, 2010).

Spread 3 and Spread 4 (1-Year ARM-Treasury and 5-Year ARM-Treasury) are the 1-year ARM Average relative to Treasury Bills and the 5-year 5/1 Hybrid ARM relative to Treasury Notes, respectively. They are representative of sub-prime rates charged on (innovative, before the financial crisis) mortgage contracts and capture the strains more directly associated to the real estate market. They can be seen as proxies of a mortgage default risk premium. The sample for Spread 3 spans the period between the week of September 20, 2002 and the week of January 1, 2016 (a total of 694 weekly observations). The sample for Spread 4 spans the period between the week of January 7, 2005 and the week of April 20, 2018 (a total of 694 weekly observations).

Spread 5 (5-Year Aaa Private-Label CMBS-Treasury) is the 5-year Aaa private-label CMBS relative to Treasury Bonds. It captures the freezing of the underlying spot market between the summer of 2007 and early 2009 (Gorton (2009b), later reversed, at least partially, thanks to the contribution of the Term Asset-Backed Securities Loan Facility (TALF) program (which began operation in March 2009 and was closed for new loan extensions on June 30, 2010, with the final outstanding TALF loan being repaid in full in October 2014). It represents the risk-premium on private-label securitized mortgages, which

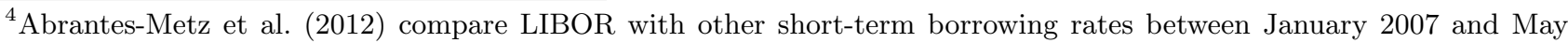
2008. They report some anomalous individual quotes, but eventually conclude that their evidence is inconsistent with a material manipulation of the U.S. dollar 1-month LIBOR. Kuo et al. (2012) show that LIBOR survey responses broadly track alternative measures of borrowing rates. Fouquau and Spieser (2015) find, instead, some evidence of manipulation. See also Duffie and Stein (2015) for additional findings. We acknowledge that evidence is mixed. In our analysis, we limit the use of the LIBOR to the computation of only one of the eight spreads.
} 
were often blamed as the root of the real estate crisis. However, this spread was not directly affected by Quantitative Easing or other policy programs. The sample for this spread extends between the week of September 20, 2002 and the week of July 19, 2013 (a total of 566 weekly observations).

Spread 6 (20-Year Moody's Baa-Aaa-Rated Corporate) is the 20-year Moody's Baa-rated corporate bonds relative to Aaa-rated corporate rate. It is a traditional indicator of credit risk, discussed extensively in the literature, also because of its forecasting power for many asset returns (Bianchi and Guidolin, 2014). It is a corporate default spread, never directly affected by Quantitative Easing or other liquidity programs during the Great Financial Crisis. The sample for Spread 6 includes the weeks between September 20, 2002 and the week of October 7, 2016 (a total of 734 weekly observations).

\section{Spread 7 (20-Year Bloomberg Fair Value U.S. Dollar Composite Bbb-Aa-Rated Corpo-} rate) is the 20-year Moody's Bbb-rated corporate relative to Aa-rated corporate bond yield (also called junk spread). It is similar to Spread 6, but it refers to riskier bonds and was rarely directly affected by policy interventions in the United States. Both Spread 6 and Spread 7 provide information about the cost of funding for businesses and therefore represent a direct measure of strains in the private non-financial sector. The sample for Spread 7 covers the period between the week of September 20, 2002 and the week of March 30, 2012 (a total of 498 weekly observations).

Spread 8 (30-Year Freddie Mac Conventional Fixed-Rate MBS-Treasury) is the 30-year Freddie Mac conventional fixed-rate MBS relative to the Treasury Bond yield. It tends to capture the credit risk of more conventional mortgage products, being representative of the premium on agency mortgage-backed securities. It was affected by the Large-Scale Asset Purchases (with short-term interest rates at nearly zero, the Federal Reserve made a series of large-scale asset purchases between late 2008 and October 2014) and Quantitative Easing programs during the financial crisis. 5 Spread 8 extends between the week of September 20, 2002 and the week of October 7, 2016 (734 weekly observations).

As they are mostly computed from Treasury or corporate yields on assets with low risk, these spreads reflect the credit-risk and (il)liquidity factors embedded in fixed income markets. Thus, the application of our research design on these spreads allows us to study and characterize any explosive behaviors in the price of credit risk plus the cost of illiquidity in each market 6 Generally positive and large skewness is associated with a sizeable divergence between mean and median in most spreads. Large excess kurtosis

\footnotetext{
${ }^{5}$ We use yield spreads from two portfolios of securities related to real estates for which the construction of sufficiently long time series is possible. Data for other mortgage rates are also available, among which a 5-year index of private-label Aaa-rated fixed-rate CMBS yields, computed by Bloomberg/Morgan Stanley; an index of 30-year fixed-rate residential prime mortgage rates computed by Freddie Mac; and a portfolio index series for lower-rated private-label MBS and CMBS yields. However, these additional time series are too short to be meaningfully used within the econometric framework that we adopt.

${ }^{6}$ For convenience, a synthetic description of each spread is provided in Table B1. Their empirical distributions are reported in Figure B1. Table B1 and Figure B1 are both available in an online Appendix.
} 
appears in all spreads, but Spreads 3 and 8. Spreads 1-8 are plotted in the lower panels of Figure 3.

A feature common to all spreads in our dataset is a substantial and synchronized increase approximately located in the middle of the sample. All spreads peak around September 2008. Such a simultaneous increase likely depends on a common factor and is, broadly speaking, the reflection of turbulence in financial markets, which would later become the Great Financial Crisis. Except for Spread 3, all spreads remain relatively flat between the beginning of the sample and 2007. Many of them are, in fact, close to their historical means (Spreads 2, 4, 6, and 7) or generally fluctuate either slightly above (Spread 8) or slightly below (Spreads 1, 3, and 5) their respective means. All spreads start widening in 2007, during the initial stages of the Great Financial Crisis, when the economic and financial turmoil only appeared to affect markets directly connected to the sub-prime real estate industry (see At-Sahalia et al., 2012). The eight interest rate spreads start rising well before Fall 2008 - i.e., the period often considered (maybe incorrectly) to mark the official beginning of the Great Financial Crisis. During the crisis, the relative increases in the spreads versus their pre-crisis levels range wildly. However, proportionally, they tend to be milder for spreads with longer maturities. As observed in an unreported investigation of several subperiods, variances and interquartile ranges show remarkable increments during the Great Financial Crisis and return close to pre-crisis levels in the months after June 2009.

\section{Results}

Table 2 reports the individual outcomes of the recursive right-tail $A D F$ tests. We resort to the Schwartz Information Criterion to select the optimal lag length in all test regressions. In each case, we allow for a maximum of 13 lags, - i.e., about three months of weekly observations $7^{7}$

We find evidence of mildly explosive behavior in all spreads but Spread 4 (5-Year ARM-Treasury) and Spread 8 (30-Year Freddie Mac Conventional Fixed-Rate MBS-Treasury). The periods over which we identify such explosive dynamics (represented by conventional grey bars) are graphically depicted in Figure 3, in Figure 4, and, using a slightly different graphical representation, in Figure 5. In the upper panels of Figure 3, we plot the sequences of spread-specific $B S A D F$ test statistics and their corresponding sequences of critical values. Some of the periods of mildly explosive behavior that we estimate are associated with generally increasing yield spreads (i.e., the price of the risky asset is declining relative to the price of the safer asset in the spread); some other estimated periods are, instead, associated with generally decreasing yield spreads (i.e., the price of the risky asset is rising relative to the price of the safer

\footnotetext{
${ }^{7}$ The time series of Spreads 1 and 2 have the same length in the sample. As such, they share the same simulated critical values. The same applies to Spreads 3 and 4, as well as Spreads 6 and 8. The tests on Spreads 5 and 7 are based on different and spread-specific critical values, as their corresponding time series span shorter periods of time.
} 
asset used to compute the spread). Specifically, we identify the periods of instability that we summarize in Table 3, where we also report an indicator of the general behavior of each spread - an increasing (I) or decreasing (D) pattern - in each estimated time frame.

Figure 4 shows the evolution of the individual interest rates from which the yield spreads in the sample are derived. The peaks of instability in the dynamics of each spread, which occur in correspondence of the global maxima in the sequences of $B S A D F$ test statistics, all appear in periods during which yield spreads tend to increase, as emphasized in Figure 5: August 31, 2007 (Spread 1, 3-Month LIBOR-OIS); October 19, 2007 (Spread 2, 3-Month ABCP-Treasury); March 21, 2008 (Spread 3, 1-Year ARM-Treasury); March 7, 2008 (Spread 5, 5-Year Aaa Private-Label CMBS-Treasury); November 21, 2008 (Spread 6, 20-Year Moody's Baa-Aaa-Rated Corporate); and December 19, 2008 (Spread 7, 20-Year Moody's Bbb-Aa-Rated Corporate). In other words, unstable dynamics peak in the U.S. fixed income markets between August 2007 and December 2008. These peaks move sequentially from short-term funding markets to mediumand long-term markets during the crisis period. Note that peaks of instability as we have defined them and peaks in the levels of the yield spreads do not have to (and, in fact, do not) correspond.

According to Contessi et al. (2014), the Great Financial Crisis started in the Summer of 2007 (during the week ending on August 3, 2007) and ended in the early Summer of 2009 (during the week ending on June 26, 2009). Concordance between the appearance of explosive dynamics associated with generally rising spreads (around July/August 2007) and the initial period of the Great Financial Crisis is evident in the cases of Spreads 1, 2, 3, and 5 - i.e., the short- and medium-term spreads. As such, these episodes of statistically unstable dynamics in these yield spreads can possibly be seen as evidence of distress in the underlying markets. Isolated spells of explosive behavior associated with generally decreasing spreads are also detected, for prolonged periods, between 2004 and 2006 and between 2010 and 2012, at least as far as Spread 3 (1-Year ARM-Treasury) is concerned. Furthermore, short periods of what can be, then, considered quick or sudden adjustment are found during the first halves of 2004 and 2015 in Spread 6 (20-Year Moody's Baa-Aaa-Rated Corporate).

The empirical methodology seems to identify the beginning of the financial crisis in the summer of 2007, during which the 3-Month LIBOR-OIS spread (Spread 1) experiences a large increase. Incidentally, in June and July 2007, Standard \& Poor's and Moody's Investor Services downgraded over a hundred bonds backed by second-lien sub-prime mortgages, and later put 612 securities backed by sub-prime residential mortgages on a credit watch. Around the same time, Bear Stearns informed investors that it would suspend redemptions from its High-Grade Structured Credit Strategies Enhanced Leverage Fund. At the end of July, Countrywide Financial Corporation filed a Securities and Exchange Commission (SEC) warning signaling "difficult conditions" and Bear Stearns liquidated two hedge funds that had 
invested in various types of mortgage-backed securities. These events, which caused turmoil in financial markets, correspond to the first episode of mild explosiveness identified in Spread 1. The following episode of disruption in the 3-Month LIBOR-OIS spread occurs between September 19, 2008 and November 14, 2008, a period characterized by the bankruptcy filing of Lehman Brothers and the first massive wave of U.S. government interventions in the form of the Troubled Asset Relief Program (TARP), a program to purchase toxic assets from financial institutions to bolster the financial sector. A third episode of mildly explosive behavior in Spread 1, again associated with a generally increasing spread, is found more recently, between the end of July 2016 and the end of October of the same year.

Our date-stamping technique captures a spell of disruption and mildly explosive behavior between August 17, 2007 and March 14, 2008 in the time series of the second short-term spread in the sample (Spread 2, or 3-Month ABCP-Treasury spread). Fitch Ratings downgraded Countrywide Financial Corporation to $\mathrm{BBB}+$ on August 16, 2007. By that time, Countrywide had entirely borrowed the $\$ 11.5$ billion available in credit lines with other banks. During the same week, the Federal Reserve Board voted to reduce the primary credit rate by 50 basis points to $5.75 \%$, thus narrowing the difference with the Federal Open Market Committee (FOMC)'s federal funds rate target to only 50 basis points. The Board of Governors of the Federal Reserve also increased the maximum term of the primary credit borrowing to 30 days, in a move to facilitate access to liquidity by qualifying banks. Despite the fact that the date-stamping technique formally limits the mildly explosive behavior in Spread 2 to the middle of March 2008, a visual inspection of the yields that we use to construct it (Figure 4) suggests that the adjustment period in the underlying markets might have, instead, ended much later, in mid-2009. In fact, as Figure 3 shows, the sequence of $B S A D F$ test statistics for Spread 2 exceeds the corresponding sequence of critical values between the end of 2008 and the first half of 2009 , a period during which the spread is particularly large. However, the $B S A D F$ statistic fails to remain above the sequence of critical values for long enough to allow a formal detection of explosiveness, which, instead, appears again between August 12, 2016 and October 7, 2016. Incidentally, this last spell of mildly explosive behavior in Spread 2 is basically coincident with the last spell detected in Spread 1.

Two of the three spreads in the sample that most accurately track the short/medium-term economic dynamics in the real estate market (Spreads 3,4, and 5) are affected by several episodes of turbulence. The 1-Year ARM-Treasury spread (Spread 3) exhibits one long episode of mildly explosive behavior associated with an upward-sloping trajectory. This episode covers the entire period of turmoil in the housing market, as it starts in mid-August 2007 and ends in the second half of January 2010. Three spells of mildly explosive behavior associated with a downward-sloping time evolution of Spread 3 are also detected. First, we identify the period between September 24, 2004 and March 24, 2006, which is 
characterized by progressive increases in the Federal Funds Rate 8 The evolution of Spread 3 over this time frame is consistent with the general behavior of financial variables at the core of development of the housing bubble. Levitin and Wachter (2012) and Justiniano et al. (2017) argue that a disproportionate increase in the supply of housing finance between 2004 and 2006 kept mortgage interest rates particularly low relative to their risk and to other interest rates, such as those associated with safe assets. Second, we find mild explosiveness in the periods June 25, 2010 - July 29, 2011 and February 24, 2012 - March 9, 2012. Arguably, given that Spread 3, on average, fell during these two time periods, such dynamics can be interpreted as sharp adjustment following the Great Financial Crisis. In particular, the beginning of the period from June 2010 to July 2011 occurs just a few months after the Federal Reserve Board increased slightly the discount rate from $0.5 \%$ to $0.75 \%$, shortened the maximum maturity for discount window loans, and, citing continued improvement in financial market conditions, held its final Term Auction Facility (TAF) auction (March 8, 2010) $!^{9}$

The date-stamping algorithm also identifies a period of turbulence in the market of commercial mortgage-backed securities, represented in our sample by Spread 5 - i.e., the 5-Year Aaa private-label CMBS-Treasury spread. The period covering the spell of mild explosiveness that we estimate (July 20, 2007 - March 27, 2009) is associated with a long and persistent spread increase. Mild explosiveness also characterizes the 20-Year Moody's Baa-Aaa-Rated Corporate spread (Spread 6) during a short spell in the first half of 2004. These weeks are associated with a generally downward-sloping trajectory of Spread 6. Furthermore, mildly explosive behavior is detected in Spread 6 during a long period associated with an increasing spread, between March 7, 2008 (just before the collapse of Bear Stearns) and April 10, 2009, which is approximately at the end of the overall disruption in funding markets. More recently, spells of mildly explosive dynamics are detected at the beginning of 2015, a period during which Spread 6 tends to decrease; and between July 2015 and March 2016, a period during which Spread 6 tends to rise.

Spread 7 (20-Year BFV USD Bbb-Aa-Rated Corporate, or junk, spread) starts exhibiting instability around the end of 2008, arguably at the peak of the financial crisis, after the bankruptcy of Lehman Brothers, as the financial panic spread from interbank markets and the shadow banking system to the funding markets for corporations - i.e., to the "real economy." In the case of this spread, short periods of turbulence are also found between April and May 2015 and in June 2011.

While we fail to detect any mildly explosive behavior in Spread 4 (5-Year ARM-Treasury) and Spread 8

\footnotetext{
${ }^{8}$ The Federal Reserve began raising the target policy rate in the fall of 2004, after a prolonged period of accommodating monetary policy that followed the recession of 2001.

${ }^{9}$ Under the TAF, the Federal Reserve auctioned term funds to depository institutions that were already eligible to borrow under the primary credit program. All advances were fully collateralized. Each TAF auction was for a fixed amount with a rate to be determined through the auction process, subject to a minimum bid rate.
} 
(30-Year Freddie Mac Conventional Fixed-Rate MBS-Treasury), (i) the sequence of BSADF test statistics for Spread 4 exceeds the appropriate sequence of critical values between the summer of 2007 and the beginning of 2009; and (ii) the sequence of $B S A D F$ test statistics for Spread 8 exceeds critical values between the beginning of 2005 and the beginning of 2006. In both cases, these periods are associated with generally rising yield spreads. See Figure 3 for details.

Finally, we investigate the possibility of migration of explosive behavior from market to market, from a peak of instability to another, by implementing the testing strategy discussed in Section 2, All results are reported in Table 4. The global peaks in the sequences of $B S A D F$ statistics for each individual spread are reported in the notes underneath the table. We do not report a global peak for Spread 4 (5Year ARM-Treasury) nor for Spread 8 (30-Year Freddie Mac Conventional Fixed-Rate MBS-Treasury), given that, in these two cases, we fail to detect any statistically significant explosive behavior. Based on the previously described chronological appearance of these peaks, we test for migration from Spread 1 (3-Month LIBOR-OIS) to Spreads 2-3 and 5-7, for migration from Spread 2 (3-Month ABCP-Treasury) to Spreads 3 and 5-7, for migration from Spread 3 (1-Year ARM-Treasury) to Spreads 6-7, for migration from Spread 5 (5-Year Aaa Private-Label CMBS-Treasury) to Spreads 3 and 6-7, and for migration from Spread 6 (20-Year Moody's Baa-Aaa-Rated Corporate) to Spread 7. Variable $m$ in the table represents the number of weekly observations between the peak in the sequence of $B S A D F$ test statistics for the spread from which we conjecture migration and the peak in the sequence of $B S A D F$ statistics for the spread towards which migration might be occurring 10 Starting from the fourth column in the table, we report (i) the estimated slope coefficient of each test regression (as described in Section 2.3), (ii) the associated standard error and t-statistic, and (iii) the numerical value of $L(m)$ and $Z_{\beta}$ computed for different values of the parameter $a$, here allowed to vary discretely between $1 / 10$ and $1 / 3$.

We detect statistically significant migration from Spread 1 (3-Month LIBOR-OIS) to Spread 3 (1Year ARM-Treasury) and Spread 5 (5-Year Aaa Private-Label CMBS-Treasury); and from Spread 2 (3-Month ABCP-Treasury) to Spread 3 (1-Year ARM-Treasury), Spread 5 (5-Year Aaa Private-Label CMBS-Treasury), Spread 6 (20-Year Moody's Baa-Aaa-Rated Corporate), and Spread 7 (20-Year BFV USD Bbb-Aa-Rated Corporate). These findings support the notion that the tensions and turmoil that emerged in the short-term funding markets in the second half of 2007 transmitted to the medium- and long-term real estate derivatives market and corporate junk bond market as the financial crisis unfolded. This evidence is consistent with the patterns in the peaks of instability described early on, as they occur sequentially and move from short-term funding markets to medium- and long-term markets between

\footnotetext{
${ }^{10}$ When we test for migration from Spread 1 to Spread 2, from Spread 5 to Spread 3, and from Spread 6 to Spread $7, m$ is likely too small (equal to 7,2 , and 4, respectively) to produce meaningfully estimated test regression coefficients.
} 
August 2007 and December 2008, arguably the most turbulent months of the Great Financial Crisis.

\section{The Panic of 2007 Revisited}

An ABX is a credit default swap (CDS) contract that pools lists of exposures to mortgage backed securities. The ABX.HE is a set of indices that tracks credit default swaps on U.S. residential mortgagebacked securities (see Reserve Bank of Australia, 2008, Fender and Scheicher, 2009, Gorton, 2009a b, for more detailed discussions). Four groups of ABX indices were issued every six months between January 2006 and 2008. Each index tracks credit default swaps on a fixed sample of twenty residential mortgagebacked securities, based on sub-prime mortgages issued in the previous six months. Each group of indices includes five sub-indices corresponding to different rating classes of residential mortgage-backed securities, namely AAA, AA, A, BBB, and BBB-. Classes BBB and BBB- represent the ratings for the riskiest subprime mortgage loans. As of 2007, these sub-indices became closely monitored barometers for changes in U.S. sub-prime debt markets and soon came to represent a focal point for all market participants. We plot these five sub-indices for each group in the four charts of Figure 6.

The ABX.HE.06-1 indices represent the first issuance of this kind of CDS and refer to tranches of twenty residential mortgage-backed securities issued in the second half of 2005. In the rolls that were released every six months in the subsequent two years, due to the deepening of the sub-prime crisis, the number of issuances dropped so much that ABX indices could not be constructed any longer, starting from 2008. While each ABX.HE index contract was issued in a fixed notional amount in which the twenty underlying tranches were equally weighted, during the life of the contract the notional amount would decline, typically due to write-downs or pre-payments. In practice, ABX.HE indices functioned like a credit default swap allowing investors to buy or sell insurance on the underlying tranches of residential mortgage-backed securities, therefore providing both hedging and trading opportunities ${ }^{11}$ Gorton (2009a b) argue that ABX.HE indices are a precious source of information regarding the pricing of sub-prime securities in the initial phases of the Great Financial Crisis. Reportedly, investors used these indices as a reference to evaluate their holdings of real-estate-related securities. The visible contraction of all these indices in 2007 prompted several financial institutions to report large credit write-downs on sub-prime related securities. Gorton (2009a) considers this event the de facto beginning of the 2007-08 panic. Later analysis rationalized this episode as financial panic akin to bank runs. However, in this

\footnotetext{
${ }^{11}$ As Gorton (2009a b) point out, given that ABX.HE indices would trade based on price rather than a spread, and given that the premium rate on each index was fixed at its launch, the market prices of such indices would adjust to reflect changes either in risk aversion or in the market evaluation of the default risk related to the underlying residential mortgage-backed securities. A price reduction below par can be interpreted as an increase in the market cost of protection relative to the same cost at launch of the product.
} 
particular crisis, panic affected the shadow banking system in addition to regulated depositary banks.

We proceed to formally test Gorton (2009a b)'s conjecture that the collapse of the ABX indices in 2007 triggered a reaction in other financial markets in the United States, including fixed income markets. Such a reaction was likely determined by a signaling mechanism about the state of the market of mortgage-backed securities. Therefore, it is sensible to test for the migration of financial distress from the $\mathrm{ABX}$ to fixed income markets after using data from the first roll of ABX indices, which provides the longest time series. In particular, we apply our testing algorithm to the BBB index only, namely the ABX.HE.BBB.06-1 series. As explained in Section 2, applying our recursive methodology for the detection of mildly explosive behavior requires an initial window of observations to initialize the algorithm. Thus, the application of the technique would consume data from the weekly ABX.HE.BBB.06-1 index series through the second half of 2007, as we use a $10 \%$ initial window for the recursion and given that the index data only span the period between the week of January 19, 2006 and the week of May 15, 2015 (488 observations). In this particular instance, we also collect daily data for the ABX.HE.BBB.06-1 index between January 19, 2006 and May 18, 2018 (a total of 2,321 observations) and apply the right-tail $A D F$ test. We find significant evidence of mildly explosive behavior at the $1 \%$ level. When the index starts collapsing at the beginning of 2007, mild explosiveness peaks for the first time (a local maximum of 6.685 in the sequence of $B S A D F$ test statistics) on February 12, 2007 and for the second time (a local maximum of 7.242, the third largest in the sample,) just a few months later, on July 26, 2007. The days that Gorton (2009a) identifies as the beginning of the panic in the ABX market are also located in the last week of July 2007. Therefore, we use this week to test for panic transmission.

Going back to weekly series, we test for panic transmission to the fixed income markets represented by the yield spreads that exhibit peaks in the sequence of $B S A D F$ statistics after the week of July $27,2007$. Empirical findings (Table 5) show evidence of transmission from the ABX.HE.BBB.06-1 index to Spreads 2, 5, 6, and 7 (maybe Spread 1, too), thus statistically validating Gorton (2009a b)'s argument. Instability transmitted directly to short and medium/long segments of U.S. fixed income markets, including some of the mortgage-related and risky corporate bond markets in the sample. Panic and a mechanism of propagation are, therefore, formally detected at the onset of the Great Financial Crisis.

\section{Conclusions}

This study contributes to the understanding of the time-series behavior of yield spreads in U.S. fixed income markets between 2002 and 2018. We give special emphasis to the turbulent years of the Great Financial Crisis. Using U.S. weekly data, we construct a panel of eight spreads from a variety of traded 
instruments and yields and identify periods of mildly explosive behavior in their dynamics. Six out of these eight spreads are characterized by spells of statistically significant mild explosiveness, which, depending on the context, can be interpreted either as periods of financial turmoil and distress or periods of quick re-adjustment and shock absorption. As a matter of fact, from a temporal point of view, the spells that we identify exhibit noticeable concordance with the timeline of the Great Financial Crisis of 2007-09 12 Such dating consistency is particularly evident in the case of short-term spreads and in the case of those spreads in the sample that, more specifically, capture the risk(s) associated with the U.S. real estate market. We also find evidence of statistical instability (possibly financial or economic distress) and migration of such instability across markets. During the Great Financial Crisis, mildly explosive dynamics migrate from markets associated with short-term funding to markets represented by spreads implicit in medium- and long-term fixed income securities.

Moreover, we investigate Gorton (2009a b)'s conjecture that ABX indices may have provided critical information about the development of real estate markets just before the beginning of the crisis in 2007 and that this information may have triggered a panic that spread across financial markets. We test for migration from a spell of mildly explosive behavior in the ABX market to the segments of fixed income markets represented by the yield spreads in our sample. Indeed, between July 2007 and December 2008, we find statistical evidence of instability migration towards the markets that have been found to be affected by spells of mild explosiveness. This finding suggests that there probably exist avenues of migration of financial distress that might be amenable to policy intervention, at least to the extent to which that distress could be detected early in the data, as the described methods offer a promise to.

Of course, avenues of further extension of both methods and application would be fruitful and deserve careful exploration. In this paper, we have looked for evidence of explosiveness in the time series of yield spreads. Statistical moments are affected, in case explosive dynamics actually occur, but we have not paid any specific attention to the informational content of, for example, conditional volatilities. Although, statistically, mild explosiveness will also be reflected in the dynamics of volatility, volatility may, by itself, contain useful and additional early-warning information that could be optimally exploited. For instance, Recchioni and Tedeschi (2017) note that in their multivariate, common stochastic volatility model there is a strong correlation between estimated volatilities and instability in government bond yields. This finding might provide the basis fo the construction of an early-warning indicator of significant instabilities that complements the one that we adopt in this paper. Kürüm et al. (2018) combine a number of mathematical tools to generate early-warning signals of financial bubbles that exploit trading volume data. They show that their index effectively declines as a bubble-burst moment approaches. In this paper, we have ignored

\footnotetext{
${ }^{12}$ See, for example, https://www.stlouisfed.org/financial-crisis/full-timeline.
} 
volume data altogether, but it would be interesting to extend our analysis in this direction.

\section{References}

Abrantes-Metz, R. M., Kraten, M., Metz, A. D., and Seow, G. S. (2012). Libor manipulation? Journal of Banking \& Finance, 36(1):136-150.

Adrian, T., Covitz, D., and Liang, N. (2015). Financial stability monitoring. Annual Review of Financial Economics, 7(1):357-395.

At-Sahalia, Y., Andritzky, J., Jobst, A., Nowak, S., and Tamirisa, N. (2012). Market Response to Policy Initiatives during the Global Financial Crisis. Journal of International Economics, 87(1):162-177.

Bernanke, B. (2002). Asset-price "bubbles" and monetary policy. Remarks before the New York Chapter of the National Association for Business Economics, New York, available at http://www.federalreserve.gov/boarddocs/speeches/2002/20021015/ default.htm.

Bianchi, D. and Guidolin, M. (2014). Can Long-Run Dynamic Optimal Strategies Outperform FixedMix Portfolios? Evidence from Multiple Data Sets. European Journal of Operational Research, 236(1):160-176.

Clark, E. and Baccar, S. (2018). Modelling credit spreads with time volatility, skewness, and kurtosis. Annals of Operations Research, 262(2):431-461.

Contessi, S., De Pace, P., and Guidolin, M. (2014). How Did the Financial Crisis Alter the Correlations of U.S. Yield Spreads? Journal of Empirical Finance, 28(C):362-385.

Del Negro, M., Giannone, D., Giannoni, M., and Tambalotti, A. (2017). Safety, liquidity, and the natural rate of interest. Brookings Papers on Economic Activity.

Diamond, P. and Dybig, P. (1983). Bank runs, deposit insurance, and liquidity. Journal of Political Economy, 91(3):401-19.

Duffie, D. and Stein, J. C. (2015). Reforming libor and other financial market benchmarks. Journal of Economic Perspectives, 29(2):191-212.

Dungey, M., Fry, R., Gonzalez-Hermosillo, B., and Martin, V. (2005). Empirical modelling of contagion: A review of methodologies. Quantitative Finance, 5:9-24.

Esther, G. (2016). The outlook and monetary policy. York, Nebraska, available at https://www.kansascityfed.org/ /media/files/publicat/speeches/2016/2016-george-york-04-07.pdf.

Fanelli, V. (2017). Implications of implicit credit spread volatilities on interest rate modelling. European Journal of Operational Research, 263(2):707-718.

Faust, J., Gilchrist, S., Wright, J. H., and Zaikrajsek, E. (2013). Credit Spreads as Predictors of RealTime Economic Activity: A Bayesian Model-Averaging Approach. The Review of Economics and Statistics, 95(5):1501-1519.

Fender, I. and Scheicher, M. (2009). The Pricing of Subprime Mortgage Risk in Good Times and Bad: Evidence from the ABX.HE Indices. Applied Financial Economics, 19(24):1925-1945.

Forbes, K. and Rigobon, R. (2001). Contagion in latin america: Definitions, measurement, and policy implications. Economia Journal of the Latin American and Caribbean Economic Association, 1(2):146. 
Forbes, K. and Rigobon, R. (2002). No contagion, only interdependence: Measuring stock market comovements. The Journal of Finance, 57:2223-2261.

Fouquau, J. and Spieser, P. K. (2015). Statistical Evidence about LIBOR Manipulation: A Sherlock Holmes Investigation. Journal of Banking 83 Finance, 50(C):632-643.

Gorton, G. (2009a). Information, Liquidity, and the (Ongoing) Panic of 2007. American Economic Review, 99(2):567-72.

Gorton, G. (2009b). The Subprime Panic. European Financial Management, 15(1):10-46.

Gourio, F. (2014). Financial distress and endogenous uncertainty. Federal Reserve Bank of Chicago, manuscript.

Guidolin, M. and Tam, Y. M. (2013). A Yield Spread Perspective on the Great Financial Crisis: BreakPoint Test Evidence. International Review of Financial Analysis, 26(C):18-39.

Hayford, M. and Malliaris, A. (2005). How did the fed react to the 1990s stock market bubble? evidence from an extended taylor rule. European Journal of Operational Research, 163(1):20 - 29. Financial Modelling and Risk Management.

Hollander, H. and Liu, G. (2016). Credit spread variability in the U.S. business cycle: The Great Moderation versus the Great Recession. Journal of Banking $\&$ Finance, 67(C):37-52.

Huang, Y., Kou, G., and Peng, Y. (2017). Nonlinear manifold learning for early warnings in financial markets. European Journal of Operational Research, 258(2):692 - 702.

Justiniano, A., Primiceri, G. E., and Tambalotti, A. (2017). The mortgage rate conundrum. Nortwestern University manuscript.

Krishnamurty, A. and Muir, T. (2017). . How Credit Cycles Across a Financial Crisis . Stanford University manuscript.

Kuo, D., Skeie, D., and Vickery, J. (2012). A comparison of libor to other measures of bank. Federal Reserve Bank of New York, manuscript.

Kürüm, E., Weber, G.-W., and Iyigun, C. (2018). Early warning on stock market bubbles via methods of optimization, clustering and inverse problems. Annals of Operations Research, 260(1):293-320.

Levitin, A. and Wachter, S. M. (2012). Explaining the housing bubble. Georgetown Law Journal, 100(4):1177-1258.

Muir, T. (2017). Financial Crises and Risk Premia. The Quarterly Journal of Economics, 132(2):765-809.

Pesaran, M. H. and Pick, A. (2007). Econometric issues in the analysis of contagion. Journal of Economic Dynamics and Control, 31:1245-1277.

Phillips, P. C. B., Shi, S., and Yu, J. (2015). Testing for Multiple Bubbles: Historical Episodes of Exuberance and Collapse in the S\&P 500. International Economic Review, 56:1043-1078.

Phillips, P. C. B. and Yu, J. (2011). Dating the Timeline of Financial Bubbles during the Subprime Crisis. Quantitative Economics, 2(3):455-491.

Recchioni, M. C. and Tedeschi, G. (2017). From bond yield to macroeconomic instability: A parsimonious affine model. European Journal of Operational Research, 262(3):1116 - 1135.

Reserve Bank of Australia (2008). The abx.he credit default swap indices. In Financial Stability Report, chapter Box B. 


\section{A Tables and Figures}

Table 1: Description of Yield Spreads

\begin{tabular}{|c|c|c|c|c|c|c|}
\hline Variable & Upper Yield & Description & Lower Yield & Description & Sample & $\begin{array}{l}\text { Number of } \\
\text { Weekly } \\
\text { Observations }\end{array}$ \\
\hline Spread 1 & 3-Month LIBOR & $\begin{array}{l}\text { 3-Month London Interbank Offered Rate: } \\
\text { Based on U.S. \$ }\end{array}$ & 3-Month OIS & 3-Month U.S. Overnight Index Swap & $09 / 20 / 2002-04 / 20 / 2018$ & 814 \\
\hline Spread 2 & 3-Month $\mathrm{ABCP}$ & $\begin{array}{l}\text { 90-Day AA Unsecured Financial Asset- } \\
\text { Backed Commercial Paper }\end{array}$ & 3-Month T-bill & 3-Month Treasury Bond Yield & $09 / 20 / 2002-04 / 20 / 2018$ & 814 \\
\hline Spread 3 & 1-Year ARM & $\begin{array}{l}\text { 1-Year Adjustable Rate Mortgage Average in } \\
\text { the United States (Discontinued on } \\
01 / 01 / 2016 \text { ) }\end{array}$ & 1-Year T-bill & $\begin{array}{l}\text { 1-Year Treasury Note Yield at Constant } \\
\text { Maturity }\end{array}$ & 09/20/2002 - 01/01/2016 & 694 \\
\hline Spread 4 & 5-Year ARM & 5/1 Hybrid Adjustable Rate Mortgages: U.S. & 5-Year Treasury & $\begin{array}{l}\text { 5-Year Treasury Note Yield at Constant } \\
\text { Maturity }\end{array}$ & $01 / 07 / 2005-04 / 20 / 2018$ & 694 \\
\hline Spread 5 & $\begin{array}{l}\text { 5-Year Aaa Private-Label } \\
\text { CMBS }\end{array}$ & $\begin{array}{l}\text { Morgan Stanley U.S. Fixed Rate CMBS } \\
\text { Conduit Aaa Avg Life 5-Year (Discontinued } \\
\text { on 07/19/2013) }\end{array}$ & 5-Year Treasury & $\begin{array}{l}\text { 5-Year Treasury Note Yield at Constant } \\
\text { Maturity }\end{array}$ & 09/20/2002 - 07/19/2013 & 566 \\
\hline Spread 6 & $\begin{array}{l}\text { 20-Year Moody's Baa- } \\
\text { Rated Corporate }\end{array}$ & $\begin{array}{l}\text { Moody's Baa Corporate Bonds Yields, Based } \\
\text { on Corporate Bonds with Remaining } \\
\text { Maturities of at Least } 20 \text { Years } \\
\text { (Discontinued on 10/07/2016) }\end{array}$ & $\begin{array}{l}\text { 20-Year Moody's Aaa- } \\
\text { Rated Corporate }\end{array}$ & $\begin{array}{l}\text { Moody's Aaa Corporate Bonds Yields, Based } \\
\text { on Corporate Bonds with Remaining } \\
\text { Maturities of at Least } 20 \text { Years } \\
\text { (Discontinued on } 10 / 07 / 2016 \text { ) }\end{array}$ & $09 / 20 / 2002-10 / 07 / 2016$ & 734 \\
\hline Spread 7 & $\begin{array}{l}\text { 20-Year Bloomberg Fair } \\
\text { Value U.S. Dollar } \\
\text { Composite Bbb-Rated } \\
\text { Corporate }\end{array}$ & BFV USD Composite Bbb 20 Year & $\begin{array}{l}\text { 20-Year Bloomberg Fair } \\
\text { Value U.S. Dollar } \\
\text { Composite Aa-Rated } \\
\text { Corporate }\end{array}$ & $\begin{array}{l}\text { BFV USD Composite Aa } 20 \text { Year } \\
\text { (Discontinued on 03/30/2012) }\end{array}$ & 09/20/2002 - 03/30/2012 & 498 \\
\hline Spread 8 & $\begin{array}{l}\text { 30-Year Freddie Mac } \\
\text { Conventional Fixed-Rate } \\
\text { MBS }\end{array}$ & $\begin{array}{l}\text { Contract Interest Rates on Commitments for } \\
\text { Fixed-Rate } 30 \text {-Year Mortgages } \\
\text { (Discontinued on 10/07/2016) }\end{array}$ & 30-Year Treasury & $\begin{array}{l}\text { 30-Year Treasury Note Yield at Constant } \\
\text { Maturity }\end{array}$ & $09 / 20 / 2002-10 / 07 / 2016$ & 734 \\
\hline
\end{tabular}

Notes. In this table, we describe how each spread is constructed and also provide spread-specific sample information. Each spread is derived as the difference between an upper yield and a lower yield. 


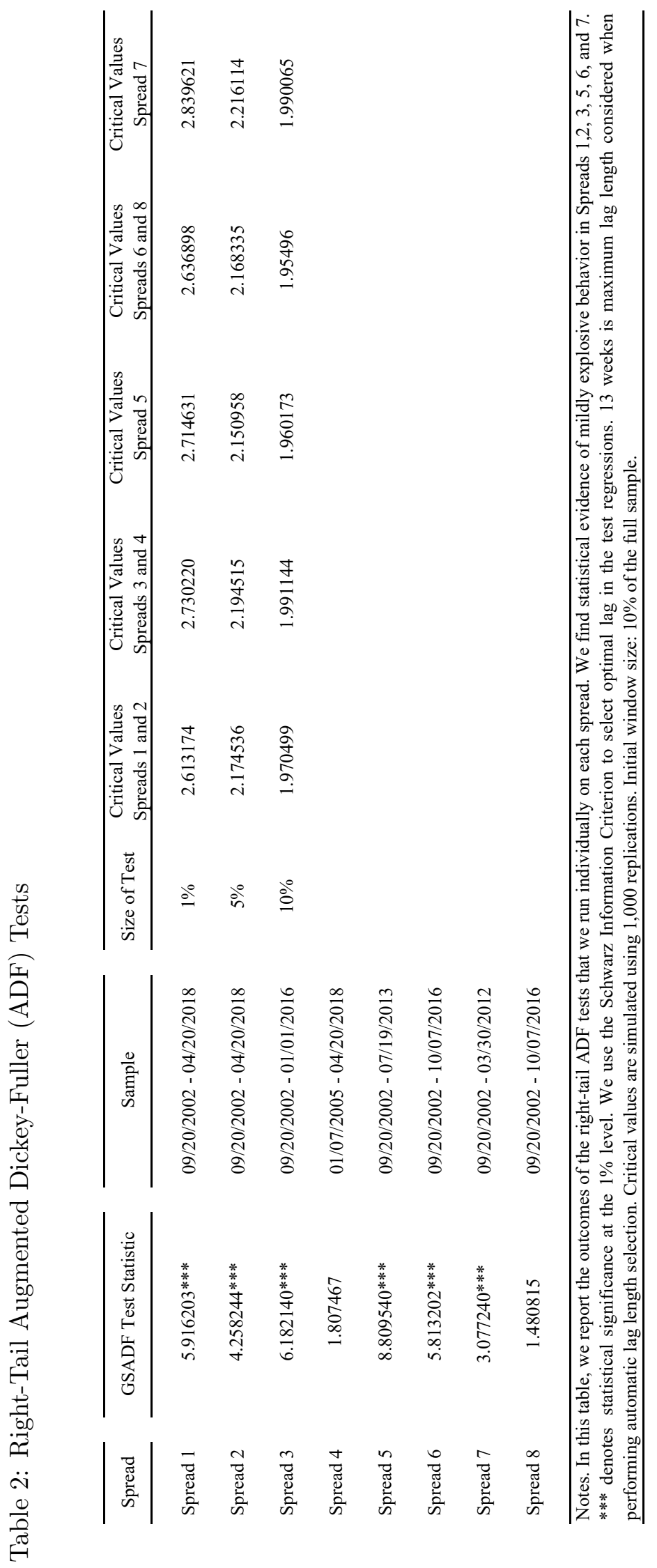


Table 3: Periods of Mildly Explosive Behavior

Spread 1

(3-Month LIBOR-OIS)

Spread 2

(3-Month ABCP-Treasury)

Spread 3

(1-Year ARM-Treasury)

\section{Spread 5}

(5-Year Aaa Private-Label CMBS-Treasury)

\section{Spread 6}

(20-Year Moody's Baa-Aaa-Rated Corporate)

\section{Spread 7}

(20-Year BFV USD Bbb-Aa-Rated Corporate)
August 3, 2007 - December 14, 2007 I

September 19, 2008 - November 14, 2008 I

July 22, 2016 - October 28, $2016 \quad$ I

August 17, 2007 - March 14, 2008 I

August 12, 2016 - October 7, 2016 I

September 24, 2004 - March 24, 2006 D

August 17, 2007 - January 22, 2010 I

June 25, 2010 - July 29, 2011 D

February 24, 2012 - March 9, 2012 D

July 20, 2007 - March 27, $2009 \quad$ I

February 6, 2004 - May 21, 2004 D

March 7, 2008 - April 18, 2008 I

July 4, 2008 - January 2, 2009 I

March 27, 2009 - April 10, 2009 I

January 23, 2015 - February 6, 2015 D

July 17, 2015 - March 4, 2016 I

April 15, 2005 - May 27, 2005 I

November 7, 2008 - June 5, $2009 \quad$ I

June 3, 2011 - June 24, $2011 \quad$ I

Notes. In this table, we report the periods of mildly explosive behavior that we estimate for each spread. Furthermore, we indicate whether those estimated time intervals are associated with generally increasing or generally decreasing yield spreads. I: generally increasing yield spread. D: generally decreasing yield spread. Peaks of mildly explosive behavior: Spread 1, 08/31/2007; Spread 2, 10/19/2007; Spread 3, 03/21/2008; Spread 5, 03/7/2008; Spread 6, 11/21/2008; Spread 7, 12/19/2008. 


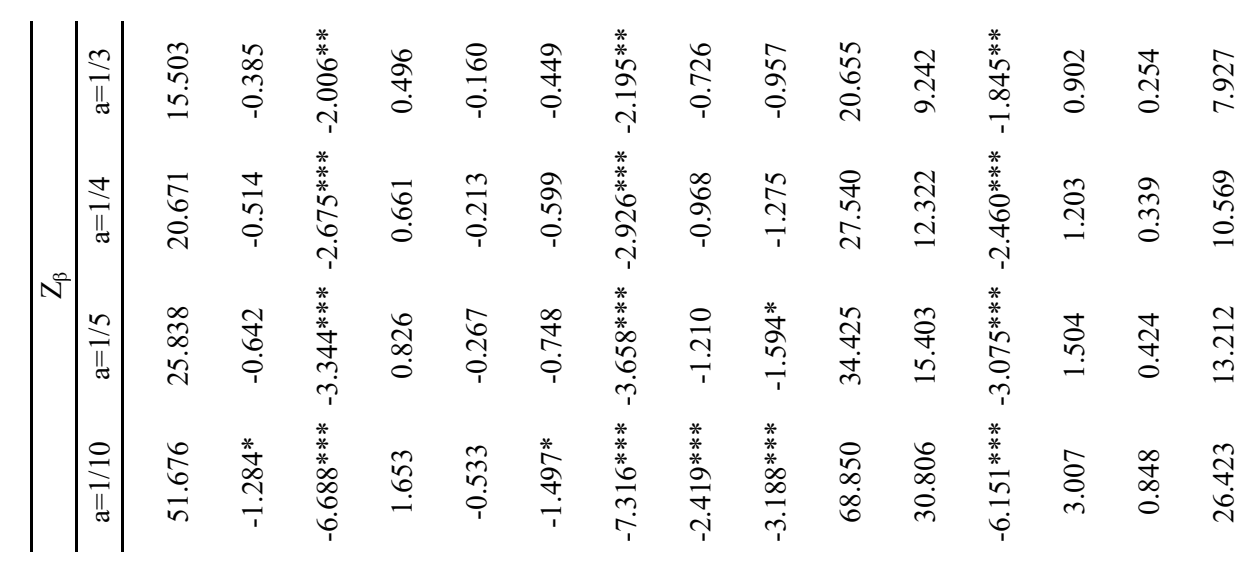

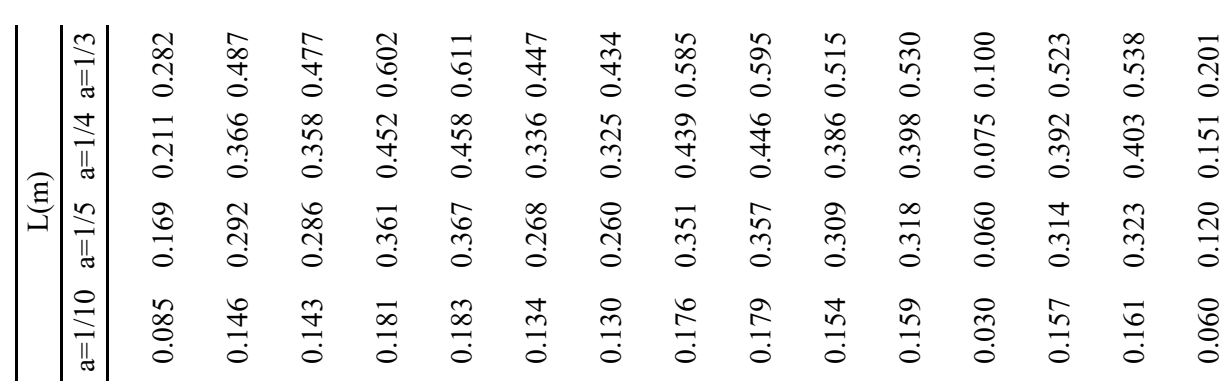

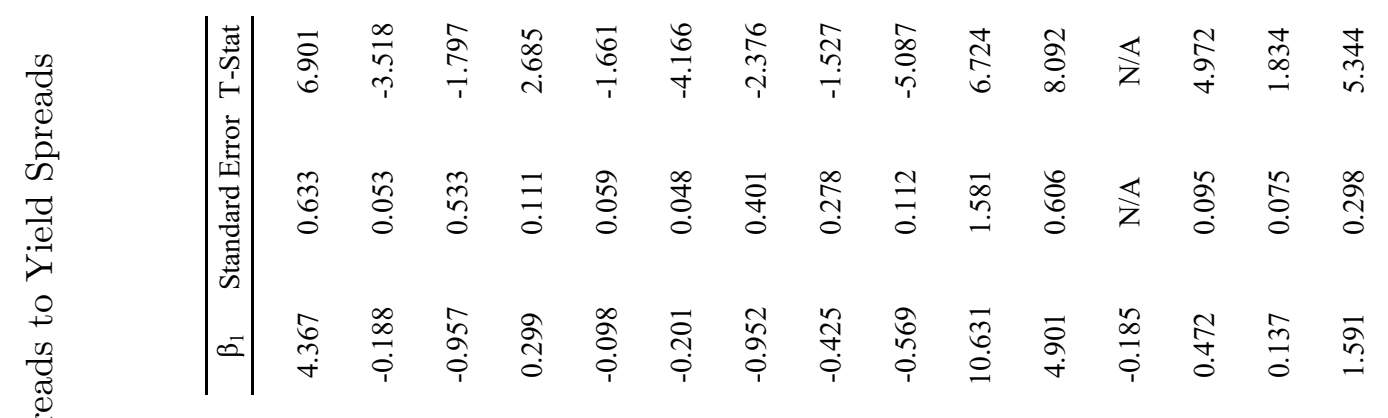$$
\text { 妾 } \mid \text { | }
$$

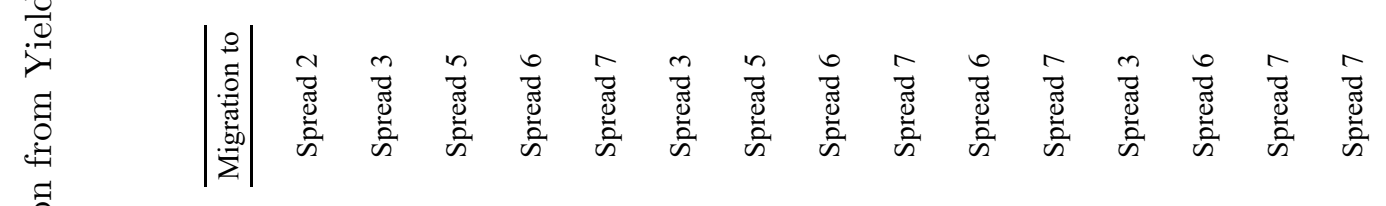

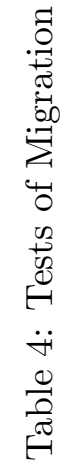

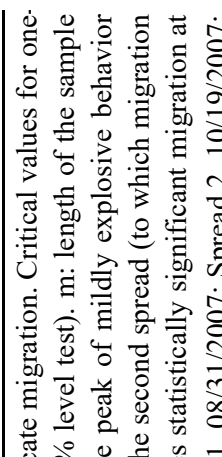

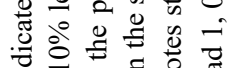

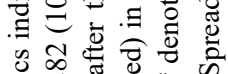

㻤

क्षे

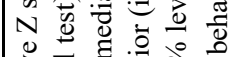

¿

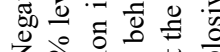

z iे 吾

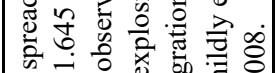

웅혈

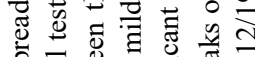

के एक क्ष

है

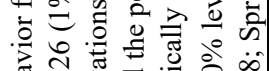

荧

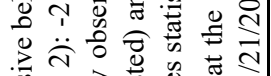

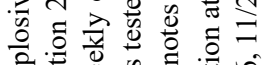

रे

중.

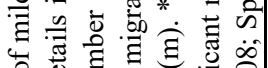

讨

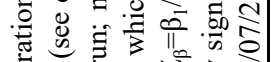

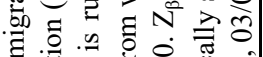

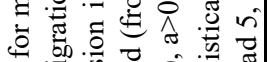

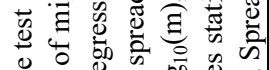

$\xi \begin{gathered}0 \\ 0\end{gathered}$

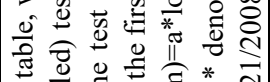

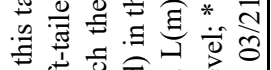

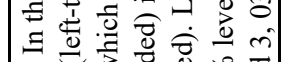

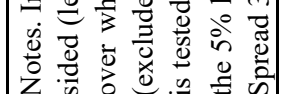




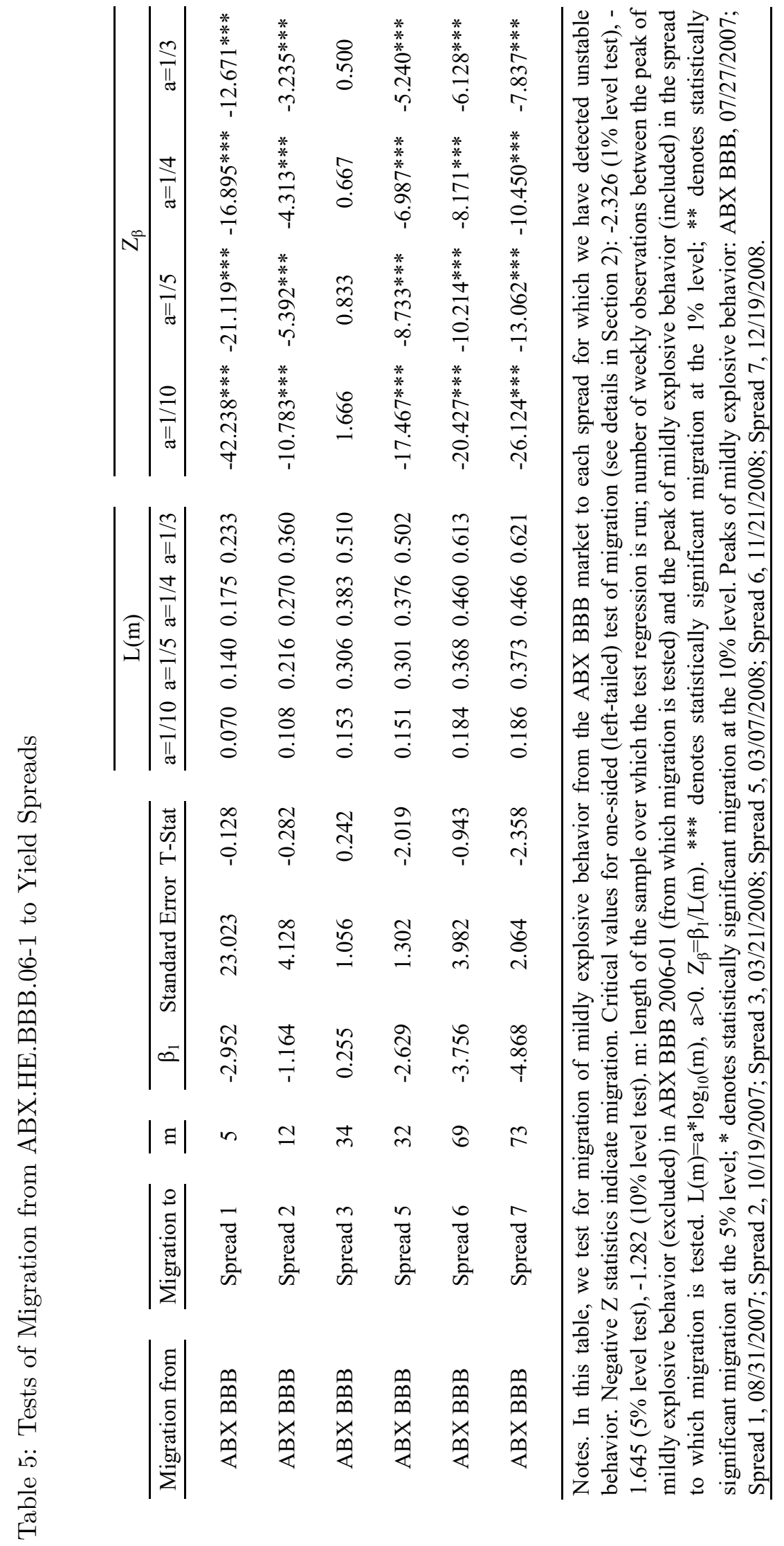


Figure 1: Mild Explosiveness, Date-Stamping, and Migration - A Diagram of the Statistical Procedure

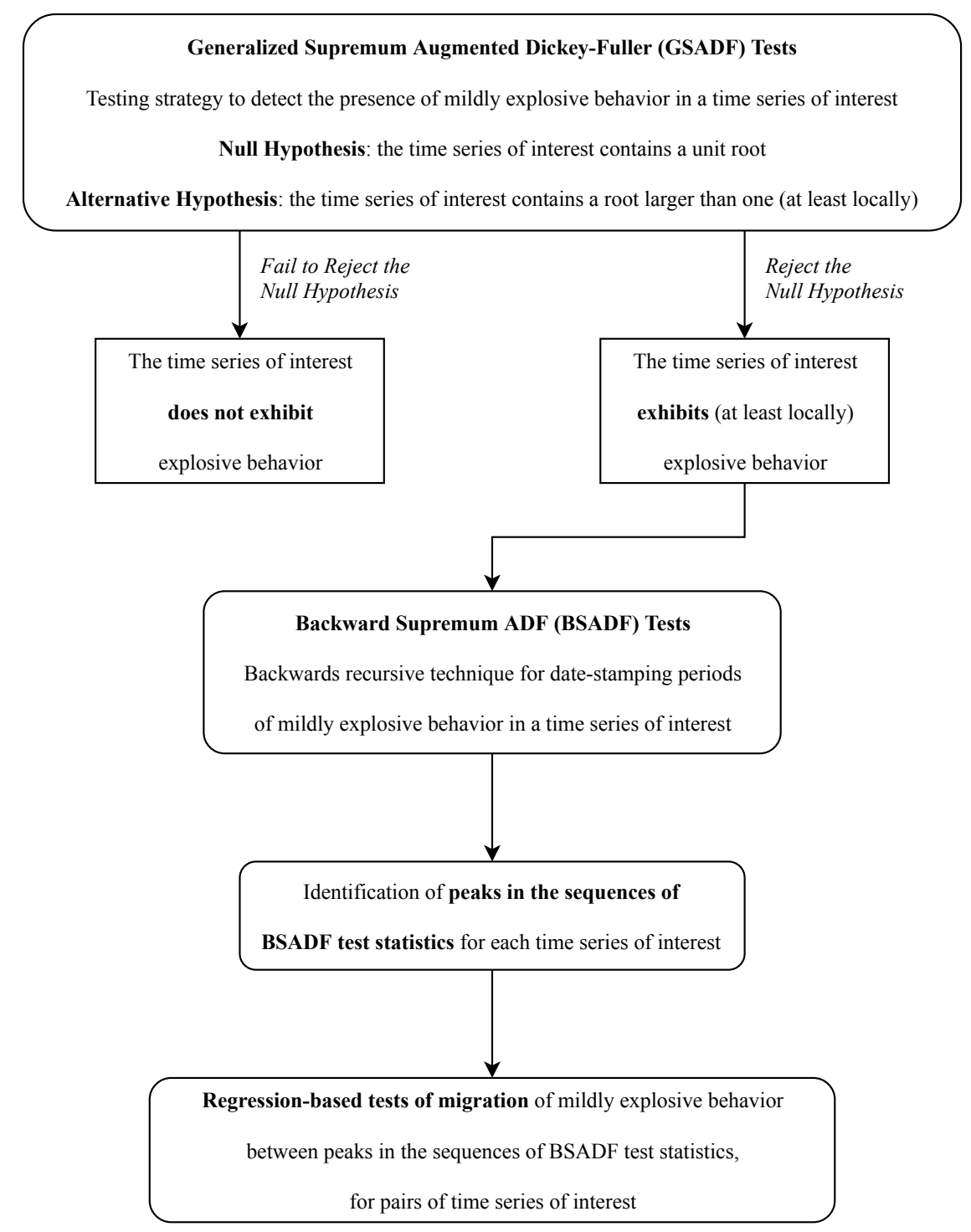

Figure 2: Recursive Mechanism for GSADF Test Statistic

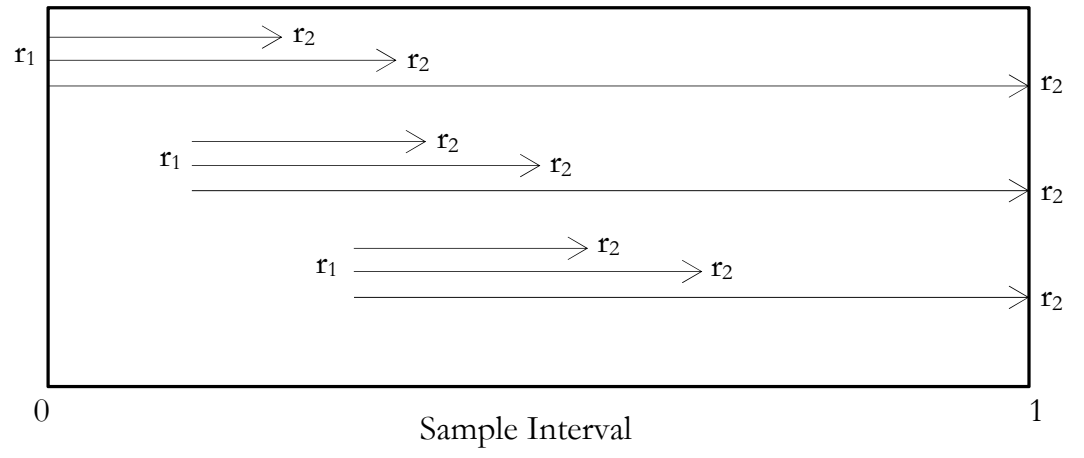


Figure 3: Yield Spreads, Sequences of BSADF Test Statistics, Sequences of Critical Values, and Periods of Mild Explosiveness

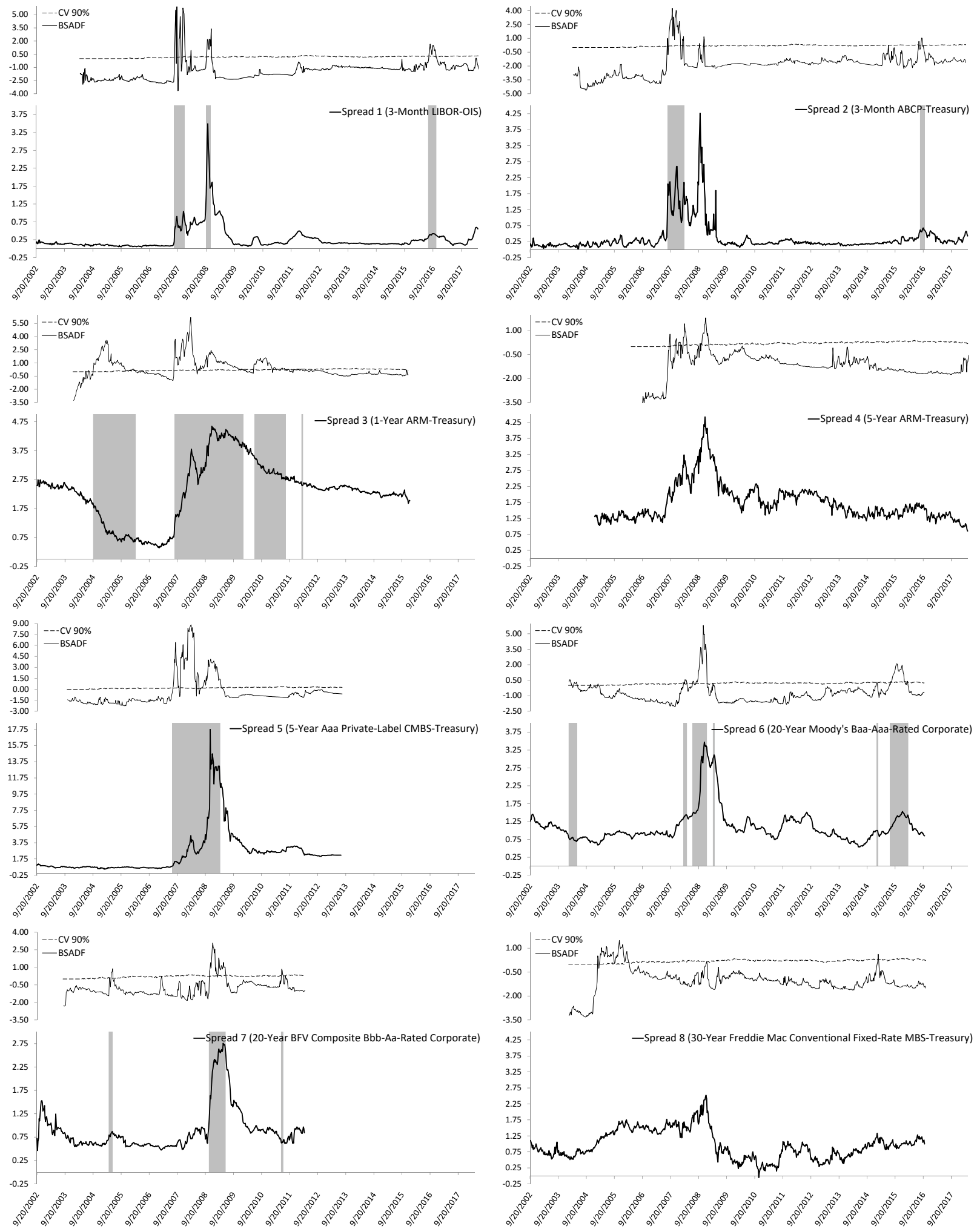

Notes. Shaded areas represent periods of mildly explosive behavior. 
Figure 4: Individual Yields for Yield Spreads Construction and Periods of Mild Explosiveness
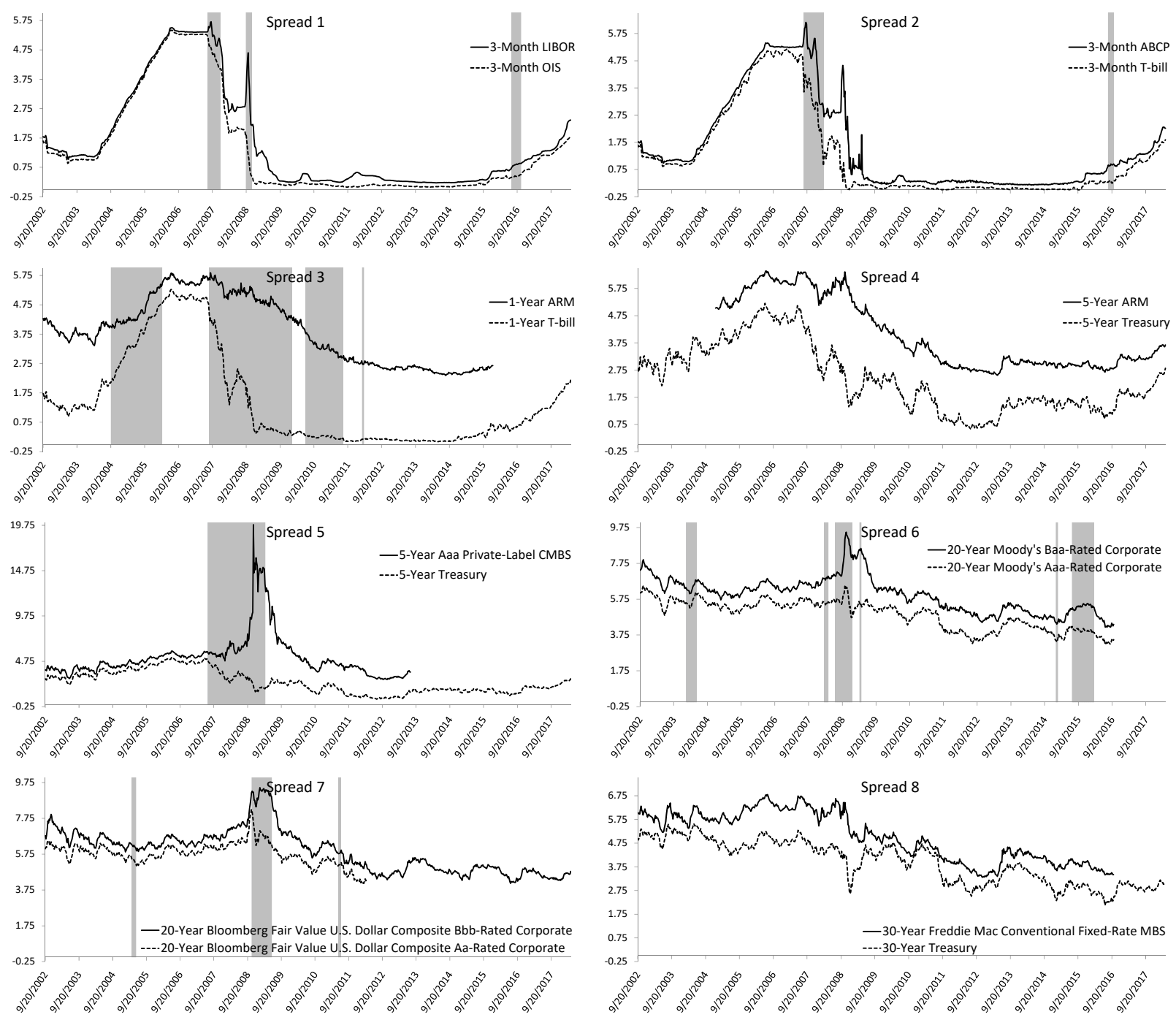

Notes. These graphs represent the yields used to construct the eight spreads described and analyzed in this paper. Shaded areas represent the periods of mildly explosive behavior that we detect in the corresponding yield spreads. 


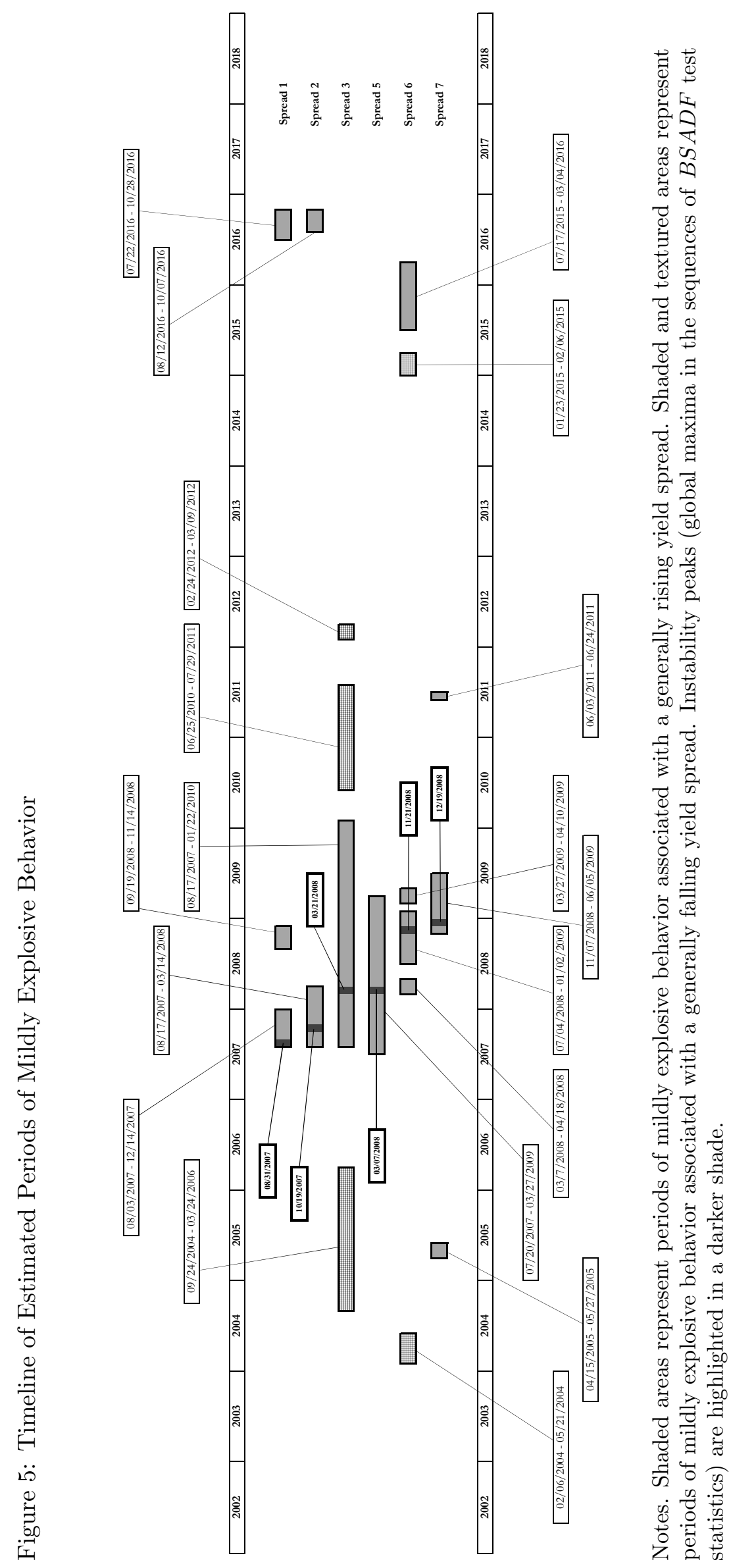




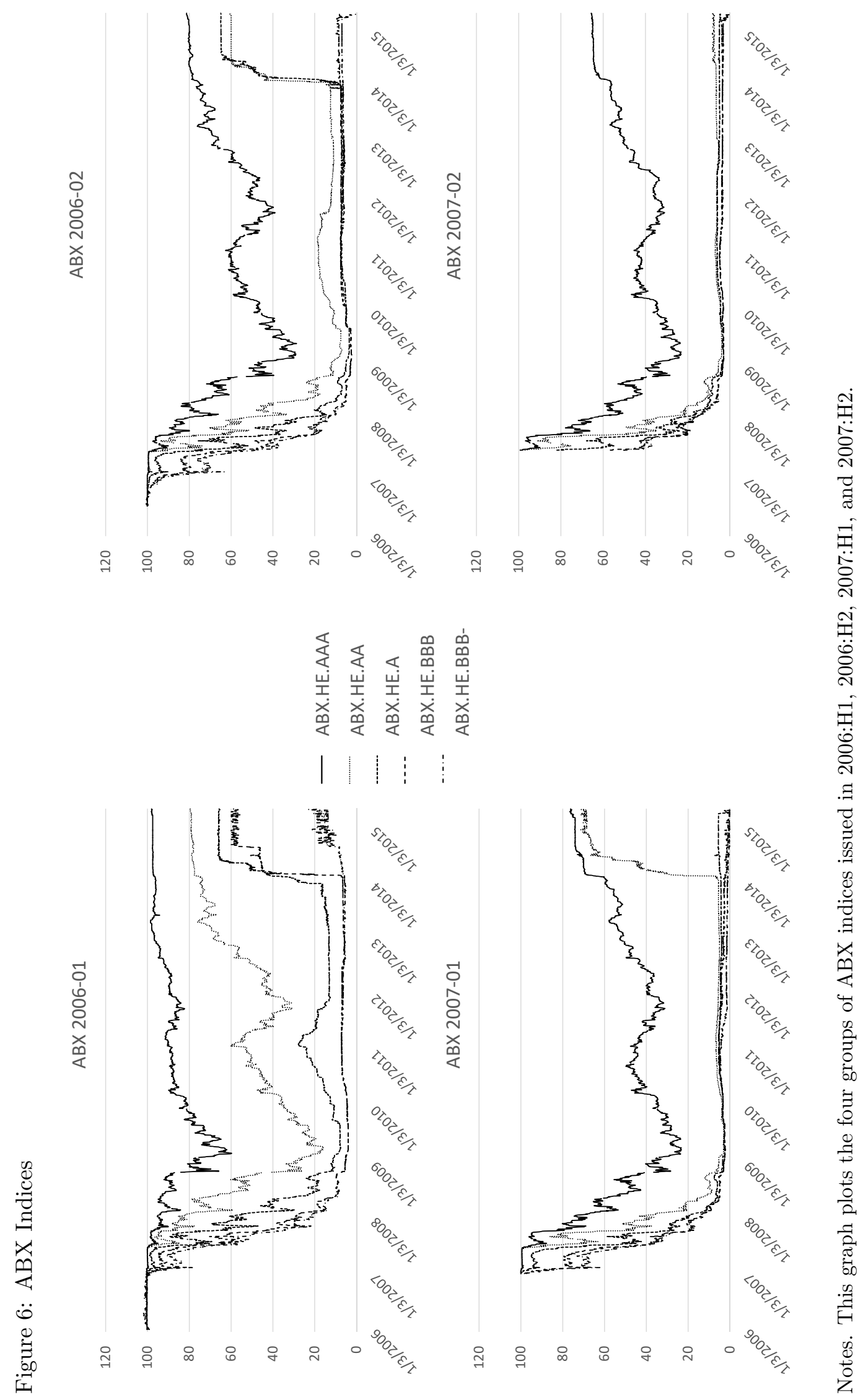

